mit dem neuen Gebiet der Chest pain Units, ein entsprechendes Register begleitet diesen neuen Versorgungsweg. Der Knackpunkt ist bei uns das Follow-up, also die Ergebnisse nach einigen Jahren, was ist aus dem Patienten geworden.

CardioVasc: Hier verlagert sich ihre Arbeit aus dem Klinikin den niedergelassenen Bereich, wo die Nachsorge stattfindet.

Senges: Dafür organisieren wir das zentrale telefonische Follow-up. Voraussetzung ist natürlich das Einverständnis des Patienten, das bereits bei der Rekrutierung eingeholt wird.

CardioVasc: Grundsätzlich haben die randomisierten Studien eine starke Patientenselektion durch die Ein- und Ausschlusskriterien. Die Register wiederum sind nicht verpflichtend, wodurch auch wieder Verzerrungen möglich werden. Wie sehen Sie diese Schwachstellen?

Senges: Ganz klar, das Rückgrat jeder klinischen Forschung ist die randomisierte Studie, die nachweist, ob eine therapeutische oder auch diagnostische Maßnahme wirksam ist. Aber genau so klar ist, die randomisierte Stu- die kann nicht alles leisten, sie ist der Wegweiser, auf dem steht: diese Maßnahme wirkt unter ganz bestimmten Bedingungen und bei ganz bestimmten Patienten. $E$ Ergänzend dazu die zweite wichtige $\checkmark$ Säule, das sind Register, um zu sehen, I wie eine Innovation umgesetzt wird:

E Wird sie überhaupt angenommen? Was bedeutet sie für die Versorgung? Lassen sich die Daten aus randomi-

sierten Studien auch im Klinikalltag reproduzieren?

Wie unterschiedlich der Studien- und Klinikalltag sein kann, hat unser MITRA-Register gezeigt, ein wichtiger praktischer Erkenntnisgewinn, den wir in der MITRA-Trias zusammengefasst haben:

- In den Registern findet man viel kränkere Patienten als in den randomisierten Studien. Ein Selektions-Bias.

- Der Nutzen jedweder Therapie, gleichgültig ob Schrittmacher, ICD oder Pharmaka, ist bei den kränkeren viel größer als bei den gesünderen.

- Schließlich das sogenannte Therapieparadox: Die kränkeren Patienten werden schlechter behandelt und die Patienten mit niedrigem Risiko bekommen die optimale, leitliniengerechte Behandlung.
ICardioVasc: Gibt es dafür eine Erklärung? Senges: Das ist noch hochspekulativ. Das Alter ist ein entscheidender Risikofaktor, deshalb wollen viele Ärzte den alten Patienten größere Interventionen gar nicht mehr zumuten. Oder aber es liegen schwere Begleiterkrankungen vor. So werden oft Betablocker nicht mehr gegeben, wenn eine COPD vorliegt, was falsch ist, denn auch Patienten mit chronischen Lungenerkrankungen und Herzinsuffizienz profitieren von Betablockern sehr.

\section{ICardioVasc: Das IHF bezieht im Mai einen} Neubau, der vermutlich auch die Möglichkeit für zusätzliche Aktivitäten bietet?

Senges: Stärker als bisher werden wir uns mit dem automatischen Daten-Export befassen, also mit Daten, die sowieso erhoben werden, die

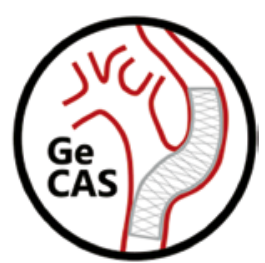
wir dann für Registerzwecke nicht doppelt zu erheben bräuchten. Die Dateneingabe kostet eine Menge Geld und wenn diese Daten aus anderen Gründen, etwa für die staatliche Qualitätssicherung, ohnehin erfasst werden, dann sollte man sie nutzen als Grundlage für weitergehende Register.

Die Fragen stellte Jochen Aumiller I

25 rekrutierende Projekte

\title{
Im Dienst der kardiologischen Versorgungsforschung
}

Was als wissenschftliches Hobby des Ludwigshafener Klinikchefs Prof. Jochen Senges begann, nämlich mit Hilfe von Registern die Versorgungsrealität in Deutschland zu dokumentieren, ist zur Zentrale der Versorgungsforschung in Deutschland gewachsen, mit Daten von über zwei Millionen Herzpatienten in ihren Registern. Das Institut für Herzinfarktforschung ging 2000 in eine Stiftung über, in der ein Team von fast 60 Mitarbeitern an der Entwicklung, Umsetzung und dem Management von prospektiven multizentrischen Registern zu verschiedenen Themen in Deutschland und Europa arbeitet.

Das IHF ist mit 200 nichtuniversitären sowie universitären Herzzentren und Abteilungen vernetzt, ebenso mit über 1000 niedergelassenen Internisten und Kardiologen in Deutschland. Wesentlichen Anstoß dazu hatte die ALKK (Arbeitsgemeinschaft leitender Kardiologischer Krankenhausärzte) gegeben. Ein wichtiges Ziel ist die Stimulierung des Qualitätswettbewerbs zwischen den Zentren. Erreicht werden soll dieser "Wettbewerb" durch die Etablierung von „fairen“ Vergleichen der Versorgungsqualität der einzelnen Zentren, allerdings setzt dies erst einmal die Akzeptanz für solche Vergleiche voraus.

Inzwischen hat das IHF die nationalen Grenzen überschritten: 20 europäische Länder nehmen an internationalen Registern des IHF teil.

Die Versorgungsforschung (outcome research) steht im Zentrum. Dazu unterhält das IHF ein Follow-up Zentrum, von dem aus monatlich ca. 3000 Pati- enten kontaktiert werden mit dem Ziel, 95\% komplette Follow-up-Datensätze von allen Patienten zu erhalten. Inzwischen gibt es kaum ein Register in Deutschland, das nicht von der Expertise des Ludwigshafener IHK profitieren wollte. Auf seiner Homepage finden sich derzeit 25 rekrutierende Projekte, darunter das Deutsche Karotisstent-Register, Deutsche TAVI-Register, Device-Register, aber auch zwei Register zur Bildgebung: das European Cardiac CT-Register und das European CMR-Register. Die Spannweite der Register umfasst die gesamte Kardiologie von der ambulanten bis zur interventionellen Innovationsforschung.

(J.A.) II

Informationen unter http://www.herzinfarktforschung.de/index.php/aktivitaeten.html 